\title{
PRÉSENCE, CHEZ DES TIQUES DE LA RÉGION DE GENÈVE, DE LARVES INFESTANTES QUI POURRAIENT SE RAPPORTER A LA FILAIRE DE CHIEN DIPETALONEMA GRASSII
}

\author{
O. BAIN*, A. AESCHLIMANN** et P. CHATELANAT***
}

RÉSUMÉ. Une quarantaine de larves infestantes de Filaire sont récoltées chez des Rhipicephalus sanguineus vivant à l'intérieur d'une habitation et importées de France ou d'Italie (2 tiques positives sur une cinquantaine observées).

Les larves appartiennent au genre Dipetalonema ; elles sont distinctes des stades infestants des 3 espèces communes en Europe, $D$. dracunculoides et $D$. reconditum du chien, $D$. rugosicauda du chevreuil. Elles pourraient par contre correspondre à D. grassii (Noé, 1907), Filaire du chien non observée depuis sa découverte en Italie.

\section{Report on infective larvae in ticks from the Geneva region which can probably be identified as the dog filarial worm, Dipetalonema grassii.}

SUMMARY. About forty infective filarial larvae were collected from Rhipicephalus sanguineus (two ticks positive out of about 50 examined) living inside a house and presumably originally transported from the south of France or Toscana. The larvae are of the genus Dipetalonema; they are distinct from infective stages of the three most common European species, $D$. dracunculoides and $D$. reconditum from dogs and $D$. rugosicauda from roe deer. They may however be larvae of $D$. arassii (Noé, 1907), a dog filarid which has not been reported since its original descovery in Italy.

I. Travail effectué dans le cadre d'un programme sur les Tiques et les maladies qu'elles transmettent, financé par le Fonds national suisse de la recherche scientifique (requète Aeschlimann, no $3.070 .8 \mathrm{I}$ ). Nous remercions Mlle S. Schneeberger, MM. O. Péter et E. Bowessidjaou ainsi que le Dr. med. vet. G. Jacquier pour leur aide technique.

* Muséum national d'Histoire naturelle, Laboratoire de Zoologie (Vers), 61 rue Buffon, F 75231 Paris Cedex 05.

** Institut de Zoologie, Chantemerle 22, 2000 Neuchâtel, Suisse.

*** 33 Moillebeau, 1209 Genève, Suisse.

Accepté le II mai r982. 
Des larves infestantes de Filaire ont été trouvées chez des Rhipicephalus sanguineus récoltés à l'intérieur d'une maison des environs de Genève à Gy (Suisse), en novembre $1981^{1}$. Sur un lot d'une cinquantaine de tiques examinées, un mâle contenait 3 larves et une femelle environ une quarantaine.

Ces larves infestantes, représentées sur la figure 1, sont longues de 1050 à $1625 \mu \mathrm{m}$ et larges de 18 à $23 \mu \mathrm{m}$; les ailes latérales sont présentes; l'œsophage est très court (220-275 $\mu \mathrm{m})$, divisé en 2 portions égales, la musculaire et la glandulaire ; la queue est longue $(65-88 \mu \mathrm{m})$ et porte à son extrémité une pointe conique ornée à sa base par 2 languettes latérales.

Une larve $\widehat{\sigma}$, longue de $1600 \mu \mathrm{m}$ et large de $21 \mu \mathrm{m}$, a les dimensions suivantes : anneau nerveux et pore excréteur à 78 et $125 \mu \mathrm{m}$ de l'extrémité céphalique ; œsophage long de $270 \mu \mathrm{m}$ et portion musculaire longue de $150 \mu \mathrm{m}$; ébauche génitale à $380 \mu \mathrm{m}$ de l'apex ; rectum long de $40 \mu \mathrm{m}$; queue longue de $88 \mu \mathrm{m}$; ailes latérales terminées à $35 \mu \mathrm{m}$ de l'extrémité caudale ; pointe caudale longue de $10 \mu \mathrm{m}$ et languettes caudales longues de $4 \mu \mathrm{m}$.

Chez une larve $q$ de $1625 \mu \mathrm{m}$ de long sur $23 \mu \mathrm{m}$ de large, anneau nerveux et pore excréteur à $78 \mu \mathrm{m}$ et $115 \mu \mathrm{m}$ de l'apex ; œsophage long de $275 \mu \mathrm{m}$ (portion musculaire longue de $117 \mu \mathrm{m}$ ) ; ébauche génitale à $175 \mu \mathrm{m}$ de l'apex ; rectum et queue longs de 42 et $78 \mu \mathrm{m}$.

Par la morphologie générale et, particulièrement, celle de l'extrémité caudale, ces larves appartiennent au genre Dipetalonema Diesing, 1861.

Il n'est connu en Europe que par un petit nombre d'espèces (4), parmi lesquelles trois sont communes : Dipetalonema (Acanthocheilonema) reconditum (Grassi, 1869), D. (A.) dracunculoides (Cobbold, 1870), tous deux parasites du chien (et autres Canidés), D. (Loxodontofilaria) rugosicauda (Böhm et Supperer, 1953), parasite du chevreuil, et récemment signalé chez Ixodes ricimus en Suisse (Aeschlimann et coll., 1979).

Les stades infestants de ces 3 espèces sont bien connus et diffèrent des larves étudiées ci-dessus.

- Les stades infestants de $D$. (A.) reconditum et $D$. (A.) dracunculoides ont, comme les adultes correspondants, un œsophage de grande taille avec une portion glandulaire très développée. De plus, les formations caudales - languette et pointe de reconditum sont arrondies (Nelson, 1962 ; Bain et Beaucournu, 1974) et les dimensions de dracunculoides sont très grandes.

- Le stade infestant de $D$. (L.) rugosicauda, dont l'adulte a un œsophage court et non divisé, possède aussi un œsophage court à petite portion glandulaire, assez semblable à celui de nos larves, mais un peu plus grand ; les dimensions du corps sont également plus grandes et les formations caudales sont pétaloïdes (Winkhardt, 1980).

La quatrième espèce, $D$. (A.) grassii (Noè, 1907), découverte chez des chiens aux environs de Rome, n'a plus été observée depuis 75 ans. Son cycle a été réalisé chez R. sanguineus (Noè, 1908), mais la morphologie du stade infestant n'est pas connue car les données publiées concernent en réalité le stade II. 

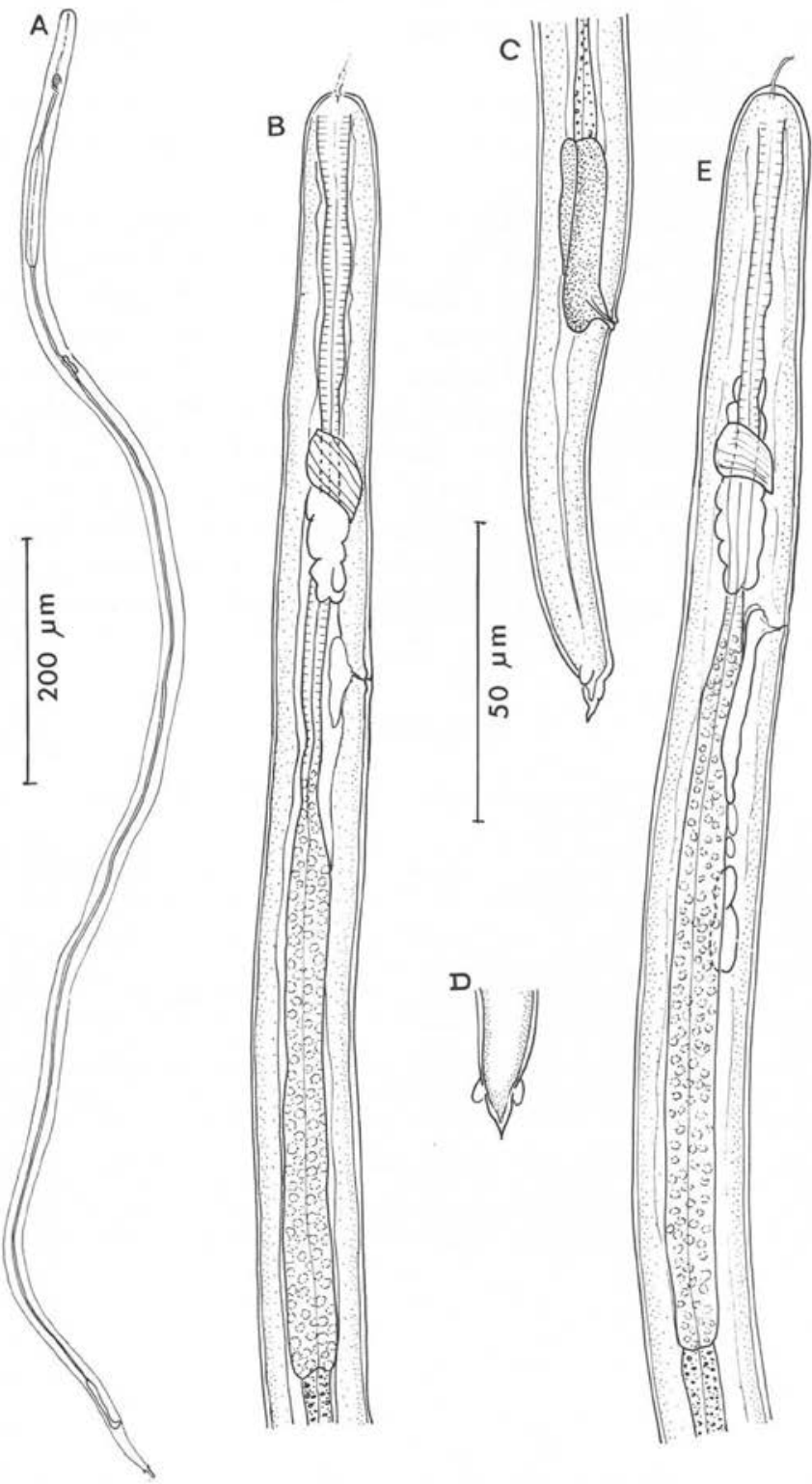

FIG. I. - Larve infestante de Dipetalonema (Acanthocheilonema) sp. chez Rhipicephalus sanguineus; A : aspect général, larve 0 ; $\mathrm{B}$ : idem, région antérieure, vue latérale* ; C : région caudale, vue latérale ; $\mathrm{D}$ : extrémité candale, vue ventrale ; $\mathrm{E}$ : région antérieure d'une larve 우, vue latérale*. (A, échelle $200 \mu \mathrm{m} ; \mathrm{B}$ à E, échelle $50 \mu \mathrm{m}$ ).

* (Chez toutes les larves, la fixation a provoqué l'extrusion de l'extrémité antérieure de l'œsophage). 
Les adultes de grassii ont une remarquable brièveté de l'œsophage avec une portion glandulaire très courte.

Cette identité de caractère avec nos larves et le fait que celles-ci ont été récoltées chez $R$. sanguineus constituent de forts arguments pour penser que ce sont des stades infestants de D. (A.) grassii.

Dans la maison où ont été récoltées les tiques filariennes, vit une petite chienne née aux États-Unis (Tennessee) en 1974, et qui a séjourné durant l'été 1975 dans le sud de la France (Région de Sainte-Maxime et Camargue) ainsi qu'en Toscane en 1976. Les deux prélèvements dermiques (région occipitale et épaule) effectués pour rechercher les microfilaires ont été négatifs, mais ce résultat n'est guère significatif.

Il est peu probable que les larves à très court œsophage, décrites ici, puissent être celles d'un Dipetalonema encore inconnu parasite d'un Mammifère sauvage, et cela d'autant plus que $R$. sanguineus n'est pas autochtone en Suisse et ne semble se maintenir qu'à l'intérieur des habitations (Aeschlimann et Büttiker, 1975).

L'hypothèse la plus vraisemblable est que la petite chienne a été contaminée par Dipetalonema (A.) grassii en France dans la région méditerranéenne ou en Italie.

\section{BIBLIOGRAPHIE}

Aeschlimann A., Büttiker W. : Importations de tiques en Suisse (Acarina : Ixodoidea). Bull. Soc. Ent. suisse, $1975,48,69-75$.

Aeschlimann A., Burgdorfer W., Matile H., PÉter O., Wyler R. : Aspects nouveaux du rôle de vecteur joué par Ixodes ricinus L. en Suisse. Acta trop., 1979, 36, I8 I-I9I.

Barn O. : Recherches sur la morphogénèse des Filaires chez l'hôte intermédiaire. Ann. Parasitol. Hum. Comp., 1972, 47, 25I-303.

Bain O., Beaucournu J. C. : Larves infestantes de Dipetalonema sp. chez des puces récoltées sur des renards du sud-ouest de la France. Ann. Parasitol. Hum. Comp., 1974, 49, 123-125.

Nelson G. S. : Dipetalonema reconditum (Grassi, 1889 ) from the dog with a note on its development in the flea, Ctenocephalides felis and the louse, Heterodoxus spiniger. J. Helm., I962, $36,297-308$.

NeLSON G. S. : Dipetalonema dracunculoides (Cobbold, I870) from the dog in Kenya ; with a note on its development in the louse fly, Hippobosca longipennis. J. Helm., 1963, 37, 235-240.

Nò̀ G. : Il ciclo evolutivo della Filaria grassii, mihi, 1907. Atti R. Accad. Lincei, Roma, 1908, $17,282-293$.

Nò̀ G. : Contribuzione alla sistematica ed alla anatomia del genere Filaria. 1. La Filaria grassii (Noè, 1907). Ricerche Lab. Anat. Norm. R. Univ. Roma (1910), I911 a, I5, 235-252.

WINKHARDT H. J. : Untersuchungen über den Entwicklungszyklus von Dipetalonema rugosicauda (syn. Wehrdikmansia rugosicauda, Nematoda : Filarioidea). Tropenmed. Parasitol., I980, 31,21 . 